\title{
An engineered food-grade Lactococcus lactis strain for production and delivery of heat-labile enterotoxin $B$ subunit to mucosal sites
}

Nan Sun ${ }^{1,2}$, Rongguang Zhang ${ }^{1,2^{*}}$, Guangcai Duan ${ }^{1,2^{*}}$, Xiaoyan Peng ${ }^{1}$, Chen Wang ${ }^{1}$, Qingtang Fan ${ }^{1}$, Shuaiyin Chen ${ }^{1}$ and Yuanlin $\mathrm{Xi}^{1}$

\begin{abstract}
Background: Recent researches have been focusing on mucosal immune adjuvants, which play the key roles in mucosal immunization and have become the limitation for non-injected vaccine development. Escherichia coli heatlabile enterotoxin B subunit (LTB) was regarded as a promising mucosal adjuvant for its nontoxicity and potent activity. LTB preparation issues have always been recurring, in part owing to that the recombinant LTB expressed by E. coli does not act as its native form.

Results: We constructed an engineered Lactococcus lactis strain using a food-grade expression system. The LTB secreted by the engineered strain was detected in the culture supernatant, constituting $10.3 \%$ of the supernatant proteins, and recognized by mouse anti-LTB antibodies. The engineered strain, co-administered orally to SPF BALB/C mice with a H. pylori vaccine candidate expressing Lpp20 antigen, could significantly enhance the Lpp20-induced mucosal SlgA antibody responses against H. pylori.

Conclusions: This is the first report that LTB was efficiently produced and delivered via using a food-grade lactococcal expression system, which offers a novel production and utilization mode of this crucial mucosal adjuvant. The engineered L. lactis strain secreting LTB has considerable potential for oral vaccine formulation owing to its outstanding safety, adjuvant activity and high-level production.
\end{abstract}

Keywords: Helicobactor pylori, Lactococcus lactis, Lpp20, Food-grade, Oral vaccine, LTB

\section{Highlights}

1. This is the first report that LTB was efficiently produced and delivered via using a food-grade lactococcal expression system.

2. This study offers a novel production and utilization mode of this crucial mucosal adjuvant.

3. The engineered $L$. lactis strain secreting LTB has considerable potential for oral vaccine formulation owing to its outstanding safety, adjuvant activity and high-level production.

\footnotetext{
* Correspondence: zrg@zzu.edu.cn; gcduan@zzu.edu.cn

${ }^{1}$ Department of Epidemiology and Statistics, College of Public Health,

Zhengzhou University, Zhengzhou 450001, China

Full list of author information is available at the end of the article
}

\section{Background}

Gastroenteric infections cause an estimated two million deaths worldwide per year, and remain severe public health issues [1,2]. As antibiotic resistance has been continually increasing, researches currently focus on developing vaccines against the causative agents, such as Helicobacter pylori, Shigella and enterotoxigenic E. coli (ETEC), for which no commercial vaccines are available [1].

Presently, most commercial vaccines are administered by parenteral routes [3]. However, recent studies demonstrated mucosal vaccination as the most effective strategy against the pathogens that colonize or invade mucosa to initiate lesions [3-5]. Although parenteral immunizations can protect against causative agents 
parasitizing host tissues via stimulation of serum antibody and cellular immune responses, they can hardly elicit mucosal immunity against noninvasive pathogens $[3,6]$. Mucosal vaccination can stimulate secretory antibody responses preventing infection by the pathogens from the mucosal surface [5]. Additionally, mucosal immunizations have the advantages of simple manipulation, less invasion, lowered risks of disease transmissions and ease of manufacture over parenteral inoculations.

However, mucosal vaccinations with antigens alone are commonly insufficient to induce marked immune responses, unless the antigens can reach the mucosal inductive sites as cholera toxins $[3,7]$. As proved, mucosal adjuvants or microbial delivery vectors are required for effective mucosal immune responses [8]. Therefore, recent researches have emphasized screening and preparation of adjuvants and the biotic delivery vehicles which possess adjuvant activity [3].

E. coli heat-labile enterotoxin B subunit (LTB) is a promising mucosal adjuvant, owing to its nontoxicity and potent mucosal adjuvant activity [9]. Nevertheless, LTB preparation issues have always been existing because it is impractical to purify LTB from ETEC for production of vaccines, and the activity of recombinant LTB (rLTB) was greatly affected by the expression hosts employed. Previous studies have indicated that preparation of LTB by using a $E$. coli expression system are not only inefficient but also costly [6]. The reasons involve the recurring formation of insoluble inclusion bodies, lower yields of bioactive rLTB, the cost of protein purification and the risk of pollution with unbeneficial bacterial components like lipopolysaccharide.

To address these issues, such bacteria as attenuated pathogens and probiotics have been exploited as expression hosts and live vectors for LTB production and delivery. A study compared expression efficacy of rLTB in Pichia pastoris with that in E. coli, demonstrating that a higher expression level and adjuvant activity of rLTB could be obtained by using $P$. pastoris as the expression host [6]. Another study proved that LTB expressed in fusion with antigens in engineered Lactobacillus can significantly enhance the local and systemic immune responses to the antigens $[10,11]$. Recently, increasing evidences supported that food-grade $L$. lactis expression systems, through expression and delivery of antigens/adjuvants, are promising oral vaccine vectors, particularly owing to their outstanding safety, avoidance of protein purification, reduced antigen degradation and efficient delivery of immunogens to the mucosal inductive sites $[12,13]$. However, successful expression of LTB in foodgrade $L$. lactis has not been reported to date. Therefore, construction of a food-grade L. lactis strain producing LTB should be a considerable step toward the goal of effective and safe mucosal vaccines.
In this work, a food-grade L. lactis strain was genetically engineered for production and delivery of LTB, and its immune adjuvant activity was evaluated by oral vaccination of mice with the engineered strain and a Lpp20-based $H$. pylori vaccine candidate. The observations of this study demonstrate a novel efficient production and utilization mode of LTB, which forms a crucial basis for mucosal vaccine formulation.

\section{Methods}

Bacterial strains, plasmids and growth conditions

The bacteria and plasmids are shown in Additional file 1 . The bacterial cultivation conditions were as described previously $[12,14]$. SPF BALB/c mice, aged 6 weeks, were purchased from Henan Experimental Animal Center (Zhengzhou, China).

\section{Polymerase chain reaction of $I t B$ gene}

The $l t B$ gene was amplified by PCR from the plasmid pMAL-c2x-mlt63 using Pyrobest DNA polymerase (TaKaRa, China). A pair of oligonucleotide primers was designed based on the published sequence (GenBank EF057802). The sequences of the primers were 5'CAGTCGGCATGCGCTCCCCAGTCTATTAC-3' (Sense) and 5'-CGCTCTAGACTAGTTTTCCATACTGATTG-3' (Antisense) with the endonuclease sites $\mathrm{Sph \textrm {I }}$ and $\mathrm{Xba \textrm {I }}$ shown in bold letters, respectively. The PCR profile included 30 cycles of $94{ }^{\circ} \mathrm{C}$ for $1 \mathrm{~min}, 55^{\circ} \mathrm{C}$ for $30 \mathrm{~s}$ and $72^{\circ}$ $\mathrm{C}$ for $2 \mathrm{~min}$.

\section{Construction of recombinant $L$. lactis strains}

The $l t B$ gene was ligated with TA cloning vector pMD19-T and used to transform E. coli DH5 $\alpha$ by heat shock. The $l t B$ gene fragment was collected by digestion of pMD19-T-ltB using SphI and XbaI (TaKaRa, China), ligated with plasmid pNZ8149-SP (GenBank KY385376), and used for transformation of L. lactis NZ3900 by electrophoration [12]. The recombinants were obtained by Elliker medium selection, and identified by restriction digestion and gene sequencing as reported before [12]. The recombinant NZ3900 strain carrying $l t B$ gene was referred to as L. lactis NZ3900/pNZ8149-SP-ltB.

\section{LTB expression and western blotting assays}

The expression of LTB in NZ3900/pNZ8149-SP-ltB was induced using $25 \mathrm{ng} / \mathrm{ml}$ nisin (Sigma, USA) as inducer under the conditions as previously described [12].

Samples of the culture supernatant were prepared from $50 \mathrm{ml}$ of culture. The supernatant was obtained by centrifugation at $10,000 \mathrm{rpm}$ for $20 \mathrm{~min}$ at $4{ }^{\circ} \mathrm{C}$, and filtered through $0.22 \mu \mathrm{m}$ filter. The proteins in the filtrate were precipitated by adding trichloroacetic acid $(10 \%, \mathrm{v} /$ v), incubating at $4{ }^{\circ} \mathrm{C}$ for $16 \mathrm{~h}$ and centrifugation at $10,000 \mathrm{rpm}$ for $30 \mathrm{~min}$ at $4{ }^{\circ} \mathrm{C}$. The pellet was 
resuspended in $8 \mathrm{ml}$ acetone, centrifugated at $10,000 \mathrm{rpm}$ for $20 \mathrm{~min}$ at $4{ }^{\circ} \mathrm{C}$, then kept in fume hood at room temperature until dry. The protein sample was added $360 \mu \mathrm{l} \mathrm{PBS}$, kept at $4{ }^{\circ} \mathrm{C}$ for $3 \mathrm{~h}$, and centrifugated at $10,000 \mathrm{rpm} 4{ }^{\circ} \mathrm{C}$ for $10 \mathrm{~min}$. The supernatant was collected and used as samples of the culture supernatant. Samples of bacterial cell lysates were processed as described before [12].

SDS-PAGE and western blotting assays were performed using mouse anti-LTB antibody (Abcam, USA) as the primary antibody as previously reported [12].

\section{Oral vaccination of mice}

The mice were assigned at random into three groups of 10 each. For Lpp20 group and Lpp $20+$ LTB group, the mice were treated by gavage with cell suspensions at a dose of $300 \mu \mathrm{l}$ of NZ3900/pNZ8149-SP-lpp20 (1× $10^{11} \mathrm{CFU} / \mathrm{ml}$ ) and a mixture of NZ3900/pNZ8149-SPlpp20 $\left(1 \times 10^{11} \mathrm{CFU} / \mathrm{ml}\right)$ and NZ3900/pNZ8149-SP-ltB $\left(1 \times 10^{11} \mathrm{CFU} / \mathrm{ml}\right)$, respectively, on day $0,7,14,21,28$ and 35. For PBS group, mice were given equal volumes of PBS instead of the cell suspensions.

\section{Blood and intestinal feces sampling}

Seven days after the last vaccination, blood and intestinal feces were collected for half number of the mice from all the groups. The blood samples were fetched from orbital sinus and kept at $4{ }^{\circ} \mathrm{C}$ for $16-20 \mathrm{~h}$, and then the sera were separated and stored in aliquots at $-20{ }^{\circ} \mathrm{C}$. For sampling intestinal feces, the mice were sacrificed by spinal dislocation, $100 \mathrm{mg}$ of feces was fetched from the intestine for each mouse, and then $1 \mathrm{ml}$ of PBS containing proteinase inhabitor (Phenylmethanesulfonyl fluoride, $0.1 \mathrm{mM}$ ) was injected by the duodenum to wash the intestinal wall. The eluate was recovered, mixed with the feces and kept at $4{ }^{\circ} \mathrm{C}$ for overnight (14-16 h). The supernatant of the mixture was separated via centrifugation at $12,000 \mathrm{rpm}$ for $10 \mathrm{~min}$, and stored at $-20^{\circ} \mathrm{C}$ as ELISA samples.

\section{ELISA detection of $\lg G$ and SIgA antibodies}

The H. pylori-specific serum IgG and fecal SIgA antibodies were quantified by ELISA as described before [12]. Briefly, 96-well microplates (Beijing Solarbio, China) were coated with soluble $H$. pylori somatic proteins. The ELISA signals were developed using biotinylated goat anti-mouse IgG (Abcam, USA), goat anti-mouse SIgA (Abcam, USA) and p-nitrophenyl phosphate (PNPP) substrate (Beijing Solarbio, China). The absorbances of the wells at $450 \mathrm{~nm}$ $\left(\mathrm{OD}_{450}\right)$ were measured using a Microplate Reader (Tecan Sunrise, $\mathrm{CH}$ ), and designed as indicators of the specific sIgA and IgG levels.

\section{Statistical analysis}

The measurement data were presented as means \pm standard deviation $(\bar{x} \pm s)$. The significance of the difference among the groups was tested using Kruskal-Wallis tests, while the pairwise comparisons of mean values were carried out using the Mann-Whitney $U$ test with the aid of software SAS9.13. The difference was considered as significant at $P<0.05$.

\section{Results}

\section{Genetic engineering of $L$. lactis}

The PCR product of $l t B$ gene was $334 \mathrm{bp}$ in length, and $100 \%$ identical in nucleotide sequence to the published E. coli ltB gene (GenBank JX504011.1). The plasmids were isolated from the $L$. lactis transformants, and identified by restriction enzyme digestion. Gene sequencing confirmed that the $l t B$ gene was exactly cloned downstream of the nisin controlled promoter (Pnis) within the expression vector pNZ8149-SP, generating pNZ8149-SP-ltB. The schematic diagram of pNZ8149SP-ltB was shown in Fig. 1.

\section{Expression and immunoreactivity of Lpp20}

To determine inducible expression of LTB in L. lactis, the cell lysates and culture supernatant were analyzed by SDS-PAGE and western blots. As results, SDS-PAGE analysis showed that a dense protein band was present at approximately $13 \mathrm{kDa}$ position in the culture supernatant of NZ3900/pNZ8149-SP-ltB, while absent at the corresponding position in the controls (Fig. 2a). The

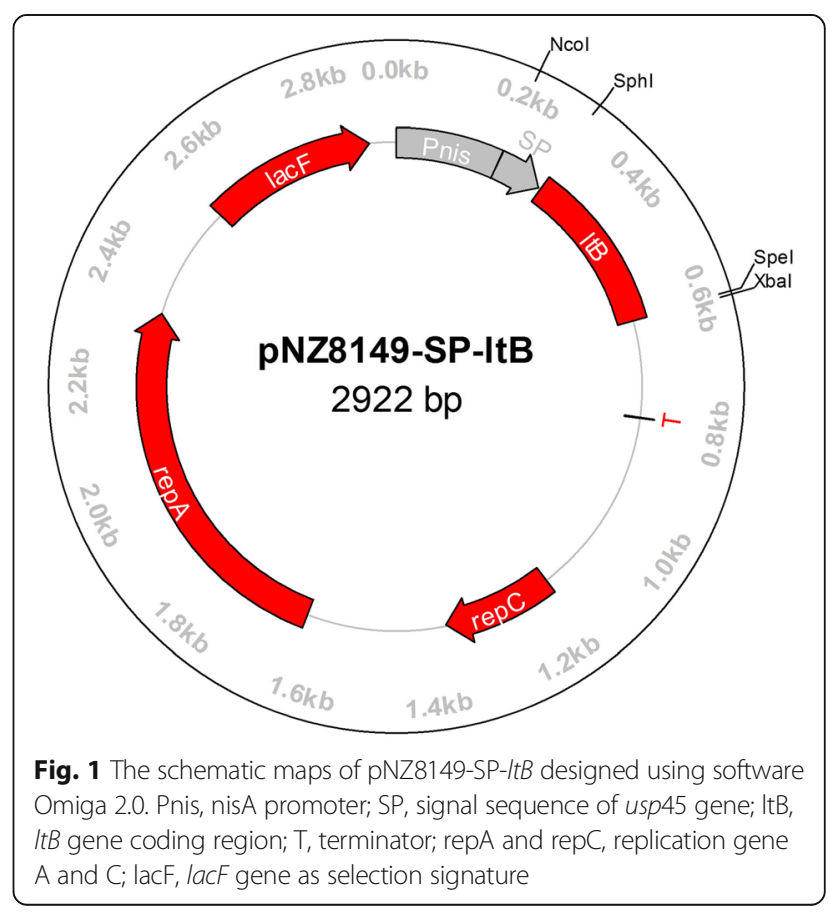



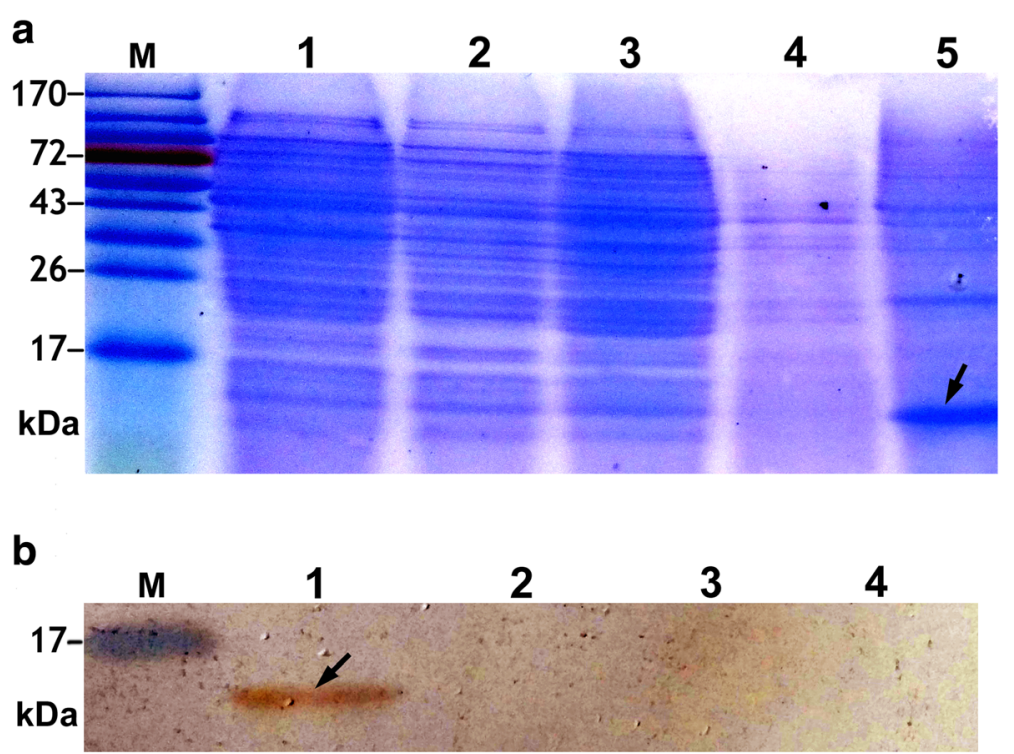

Fig. 2 Analysis of cell lysate and culture supernatant proteins of the nisin-induced L. lactis strains. The arrows indicate the expressed UreB. a SDSPAGE. Lane M, protein markers; lane 1, 2, 3, cell lysates of NZ3900, NZ3900/pNZ8149-SP and NZ3900/pNZ8149-SP-ItB, respectively; lane 4, 5, culture supernatant of NZ3900/pNZ8149-SP and NZ3900/pNZ8149-SP-ItB, respectively. These figures showed that the recombinant LTB was expressed as a secreted protein. b Western blotting assays. L. lactis NZ3900/pNZ8149-SP was used as the negative control, while mouse anti-LTB antibodies as the primary antibodies for detection of LTB. Lane M, protein markers; lane 1, 2, culture supernatant of NZ3900/pNZ8149-SP-ltB and NZ3900/ pNZ8149-SP, respectively; lane 3, 4, cell lysates of NZ3900/pNZ8149-SP-ItB and NZ3900/pNZ8149-SP, respectively. These figures showed that the expressed LTB was detectable only in the culture supernatant of NZ3900/pNZ8149-SP-ItB, and possessed immunoreactivity with the commercial anti-LTB antibody

percentage of LTB reached $10.3 \%$ in the culture supernatant proteins of the engineered stain.

Western blotting analysis showed that the $13 \mathrm{kDa}$ protein in the culture supernatant of NZ3900/pNZ8149-SP-ltB could be recognized by the mouse anti-LTB antibodies, while there was no positive band at the corresponding position in the lanes of NZ3900/pNZ8149-SP samples as negative controls (Fig. 2b).

\section{Immunization of mice and antibody assays}

The L. lactis NZ3900/pNZ8149-SP-lpp20 and NZ3900/ pNZ8149-SP- $l t B$ were induced with nisin as mentioned above. The mice of the immunized groups were orally inoculated with the induced NZ3900/pNZ8149-SP-lpp20 alone and its mixture with NZ3900/pNZ8149-SP-ltB, respectively, while the control group was treated with PBS. On day 7 after the final vaccination, the serum and intestinal feces were sampled, and tested for $H$. pylori-specific antibodies by ELISA assays. The results are shown in Additional file 2 and Fig. 3. The group treated with NZ3900/pNZ8149-SP-lpp2O alone had enhanced anti- $H$. pylori serum antibody responses, but no significantly elevated intestinal SIgA level, compared with the PBS group. The vaccination with the mixture of the two $L$. lactis strains evoked significantly elevated SIgA antibody level, and insignificantly lowered the serum IgG response, as compared with the group administered with

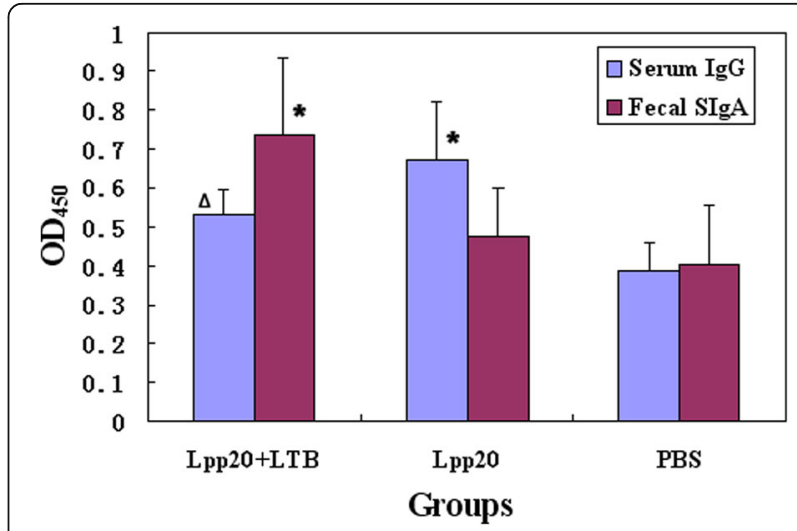

Fig. $3 \mathrm{OD}_{450}$ values of ELISA tests for $\mathrm{H}$. pylori-specific serum IgG and intestinal SlgA levels. The Lpp20 group and Lpp20 + LTB group were vaccinated with L. lactis NZ3900/pNZ8149-SP-Ipp20 and the mixture of NZ3900/pNZ8149-SP-Ipp20 and NZ3900/pNZ8149-SP-ItB, respectively, while the PBS group, as the control, was treated with PBS. One week after oral immunization, blood sampling was performed for half number of the mice of all the groups. The serum (1:10 diluted) and fecal samples were tested for $\mathrm{H}$. pylori-specific antibodies by ELISA. Bars, mean $\mathrm{OD}_{450}$ value; error bars, standard deviation; ${ }^{\triangle}$ the value was significantly higher than that for the PBS group $(P<0.05)$; ${ }^{*}$ the value was significantly different from those for the other two groups $(P<0.05)$ 
NZ3900/pNZ8149-SP-lpp20 alone. The results demonstrated the adjuvant activity of the engineered $L$. lactis expressing LTB to promote mucosal immune responses.

\section{Discussion}

As proved, the species of bacteria as expression hosts might greatly affect the bioactivity of recombinant expression products [6]. Through screening of various microbial expression systems, it is possible to establish an efficient method for production and utilization of LTB. Nevertheless, although studies have demonstrated the potential of LTB as mucosal adjuvant and accumulated evidences proved $L$. lactis to be a safe vaccine vehicle, expression and delivery of LTB using a food-grade L. lactis strain have not been reported yet. Therefore, successfully engineering a food-grade $L$. lactis strain to secret LTB can be a crucial basis for mucosal vaccine development.

L. lactis NZ3900/pNZ8149-SP is a food-grade nisincontrolled expression system, which was constructed in our previous study by introducing a gene fragment encoding the signal peptide of L. lactis Usp45 protein into pNZ8149 for capacity of secretory expression of heterologous genes [12]. In this work, the $l t B$ gene was inserted downstream of the nisin controlled promoter within pNZ8149-SP, and expression of LTB was achieved by inducement with food-grade subtoxic amount of nisin. All the components of L. lactis NZ3900/pNZ8149-SP-ltB, except for the target gene $l t B$, possess food-grade safety, suggesting the extremely high safety of the engineered strain as the LTB producer.

As reported, the L. lactis expression efficiency of heterologous protein can be rather low, and thus the expression products in certain studies were detectable by western blots, but not by SDS-PAGE $[15,16]$. In this work, the expressed LTB was visual as the predominant band in the SDS-PAGE pattern, constituting $10.3 \%$ of the culture supernatant extracts of the engineered $L$. lactis. The expression efficacy was much higher than those reported [12, 15-17]. The mechanism underlying this phenomenon might include the diversity of the heterologous genes in genetic codon constitution. The LTB expressed in L. lactis had a molecular weight of approximately $13 \mathrm{kDa}$, corresponding to that deduced from the nucleotide sequence (GenBank JX504011.1). Western blotting assays showed that LTB produced by the engineered L. lactis strain retained potent antigenicity. These findings indicate that the engineered strain culture can be a novel source of LTB, from which LTB can be obtained and utilized without protein purification and the risk of pollution with toxic components from the commonly used expression hosts, such as E. coli and Salmonella spp..
A recurring issue on heterogeneous expression is that the recombinant products might lose bioactivity of the native proteins. Although the molecular basis of LTB adjuvant properties remains unclear, it might be included that LTB interacts with such receptors as GM1-ganglioside accelerating uptake of the toxic subunit A into the epithelial cells $[6,18]$. In the present study, it was observed that vaccination of mice with L. lactis expressing Lpp20 was capable of arousing remarkable serum IgG antibody responses, but failed to induce significantly elevated intestinal specific SIgA antibody levels, as compared with the group given PBS, indicating that without the aid of suitable mucosal adjuvants, the employed $L$. lactis strain producing Lpp20 can hardly evoke notable mucosal immune responses. Similarly, a previous study showed that a $L$. lactis strain producing $H$. pylori UreB antigen was unable to elicit marked immune responses in mice [17]. The adjuvant activity of the engineered strain secreting LTB has been proved by the observations that the group received the mixture of the two engineered $L$. lactis strains expressing Lpp20 and LTB, respectively, had significantly elevated sIgA antibodies, in comparison with the group given the Lpp20-producing strain alone. Since mucosal SIgA antibodies have the key roles in immune protection against the pathogens invading human tissues by the mucosal surface, the findings of our study suggest that the engineered strain secreting LTB might markedly enhance the immune protective efficacy of the co-administered antigens [19].

Interestingly, accompanying with the enhanced mucosal SIgA, the serum IgG antibodies seemed to be suppressed in the mice immunized with the mixture of the two L. lactis strains. This result suggests that the LTBsecreting L. lactis might modulate the systemic and mucosal immune responses with opposite effects. The mechanism and its implication for vaccine designs need further investigation.

In recent decades, although in most countries the use of genetically modified organisms (GMOs) was prohibited or heavily regulated, it has been proposed to change the present regulations [20,21]. In fact, the amount of commercially available GMOs has been continuously rising in recent years [22]. It is hopeful for the food-grade $L$. lactis engineered here to be used in pharmacy and food industry in future.

\section{Conclusion}

Taken together, this is the first report that E. coli LTB was efficiently produced and delivered via using a foodgrade lactococcal expression system, which offers a novel production and utilization mode of this crucial mucosal adjuvant. The engineered $L$. lactis strain secreting LTB has considerable potential for oral vaccine formulation, owing to its outstanding safety, adjuvant activity and high-level production. 


\section{Additional files}

Additional file 1: Table S1. Bacteria and plasmids used in this work (DOC 39 kb)

Additional file 2: Table S2. $\mathrm{OD}_{450}$ values of ELISA tests for $\mathrm{H}$. pylorispecific lgG and SlgA levels. (DOC $60 \mathrm{~kb}$ )

\section{Abbreviations}

ELISA: Enzyme-linked immunosorbent assay; ETEC: Enterotoxigenic E. coli; LTB: Heat-labile enterotoxin B subunit; PBS: Phosphate buffer saline; PNPP: Pnitrophenyl phosphate; SPF: Specific pathogens free; UreB: Urease B subunit

\section{Acknowledgments}

The authors are grateful to the funding suppliers mentioned above.

\section{Funding}

This study was funded by China Postdoctoral Science Foundation (200801273) and Henan Innovation Center of Molecular Diagnosis and Laboratory Medicine (XTCX-2015-ZD2)

\section{Availability of data and materials}

All data generated during this study are included in this published article and its Additional files 1 and 2 .

\section{Authors' contributions}

NS engineered the L. lactis strain, and performed the Western blot analysis and animal experimentation. RZ and GD took the responsibility of designing and executing the project, and writing the manuscript. RZ, XP, CW and SC performed the immunization of mice and evaluation of immune efficacy. QF and $Y X$ contributed to the animal experiments. All authors read and approved the final manuscript.

\section{Competing interests}

The authors declare that they have no competing interests.

\section{Consent for publication}

Not applicable.

\section{Ethics approval}

The animal experimentations were approved by the Institutional Review Board at Zhengzhou University (no reference number), and performed complying with the ARRIVE guidelines. This study does not involve human participants.

\section{Author details}

'Department of Epidemiology and Statistics, College of Public Health Zhengzhou University, Zhengzhou 450001, China. ${ }^{2}$ Henan Innovation Center of Molecular Diagnosis and Laboratory Medicine, Xinxiang Medical University, Xinxiang 453003, China.

Received: 18 August 2016 Accepted: 1 March 2017

Published online: 06 March 2017

\section{References}

1. Daryani A, Hosseini-Teshnizi S, Hosseini SA, Ahmadpour E, Sarvi S, Amouei $A$, et al. Intestinal parasitic infections in Iranian preschool and school children: A systematic review and meta-analysis. Acta Trop. 2017;169:69-83.

2. Black RE, Cousens S, Johnson HL, Lawn JE, Rudan I, Bassani DG, et al. Global, regional, and national causes of child mortality in 2008: a systematic analysis. Lancet. 2010;375:1969-87.

3. Pérez O, Batista-Duharte A, González E, Zayas C, Balboa J, Cuello M, et al. Human prophylactic vaccine adjuvants and their determinant role in new vaccine formulations. Braz J Med Biol Res. 2012;45:681-92.

4. Holmgren J, Bourgeois L, Carlin N, Clements J, Gustafsson B, Lundgren A, et al. Development and preclinical evaluation of safety and immunogenicity of an oral ETEC vaccine containing inactivated E. coli bacteria overexpressing colonization factors CFA/I, CS3, CS5 and CS6 combined with a hybrid LT/CT B subunit antigen, administered alone and together with dmLT adjuvant. Vaccine. 2013;31:2457-64.
5. Lundgren A, Jertborn M, Svennerholm AM. Induction of long term mucosal immunological memory in humans by an oral inactivated multivalent enterotoxigenic Escherichia coli vaccine. Vaccine. 2016;34:3132-40.

6. Ma X, Yao B, Zheng W, Li L. Comparative study on characterization of recombinant $B$ subunit of $E$. coli heat-labile enterotoxin (rLTB) prepared from E. coli and P. patoris. J Microbiol Biotechnol. 2010;20:550-7.

7. Norton EB, Branco LM, Clements JD. Evaluating the A-subunit of the heatlabile toxin (LT) as an immunogen and a protective antigen against enterotoxigenic Escherichia coli (ETEC). PLoS One. 2015;10:e0136302.

8. Kataoka K, Fujihashi K. Dendritic cell-targeting DNA-based mucosal adjuvants for the development of mucosal vaccines. Expert Rev Vaccines. 2009:8:1183-93.

9. Jawale CV, Lee JH. Salmonella enterica serovar enteritidis ghosts carrying the Escherichia coli heat-labile enterotoxin B subunit are capable of inducing enhanced protective immune responses. Clin Vaccine Immunol. 2014;21:799-807.

10. Yu Q, Zhu L, Kang H, Yang Q. Mucosal Lactobacillus vectored vaccines. Hum Vaccin Immunother. 2013:9:805-7.

11. Yu M, Qi R, Chen C, Yin J, Ma S, Shi W, et al. Immunogenicity of recombinant Lactobacillus casei-expressing F4 (K88) fimbrial adhesin FaeG in conjunction with a heat-labile enterotoxin A (LTAK63) and heat-labile enterotoxin B (LTB) of enterotoxigenic Escherichia coli as an oral adjuvant in mice. J Appl Microbiol. 2017;122:506-15.

12. Chen SY, Zhang RG, Duan GC, Shi JX. Food-grade expression of Helicobacter pylori ureB subunit in Lactococcus lactis and its immunoreactivity. Curr Microbiol. 2011;62:1726-31.

13. Wyszyńska A, Kobierecka P, Bardowski J, Jagusztyn-Krynicka EK. Lactic acid bacteria-20 years exploring their potential as live vectors for mucosal vaccination. Appl Microbiol Biotechnol. 2015;99:2967-77.

14. Ma YJ, Duan GC, Zhang RG, Fan QT, Zhang WD. Mutation of iceA in Helicobacter pylori compromised IL-8 induction from human gastric epithelial cells. J Basic Microbiol. 2010;50:583-8.

15. Zhang XJ, Feng SY, Li ZT, Feng YM. Expression of Helicobacter pylori hspA gene in Lactococcus lactis NICE system and experimental study on its immunoreactivity. Gastroenterol Res Pract. 2015;2015:750932.

16. Gu Q, Song D, Zhu M. Oral vaccination of mice against Helicobacter pylori with recombinant Lactococcus lactis expressing urease subunit B. FEMS Immunol Med Microbiol. 2009;56:197-203.

17. Lee MH, Roussel Y, Wilks M, Tabaqchali S. Expression of Helicobacter pylori urease subunit B gene in Lactococcus lactis MG1363 and its use as a vaccine delivery system against H. pylori infection in mice. Vaccine. 2001:19:3927-35.

18. Wang B, Pan X, Wang H, Zhou Y, Zhu J, Yang J, et al. Immunological response of recombinant $H$. pylori multi-epitope vaccine with different vaccination strategies. Int J Clin Exp Pathol. 2014;7:6559-66.

19. Wang S, Liu H, Zhang X, Qian F. Intranasal and oral vaccination with protein-based antigens: advantages, challenges and formulation strategies. Protein Cell. 2015;6:480-503.

20. McHughen A. A critical assessment of regulatory triggers for products of biotechnology: Product vs. process. GM Crops Food. 2016;7:125-58.

21. Korobko IV, Georgiev PG, Skryabin KG, Kirpichnikov MP. GMOs in Russia: research, society and legislation. Acta Naturae. 2016;8:6-13.

22. Peng $C$, Wang $P, X u X$, Wang $X$, Wei $W$, Chen $X$, et al. Development of a qualitative real-time PCR method to detect 19 targets for identification of genetically modified organisms. Springerplus. 2016;5:889.

\section{Submit your next manuscript to BioMed Central and we will help you at every step:}

- We accept pre-submission inquiries

- Our selector tool helps you to find the most relevant journal

- We provide round the clock customer support

- Convenient online submission

- Thorough peer review

- Inclusion in PubMed and all major indexing services

- Maximum visibility for your research

Submit your manuscript at www.biomedcentral.com/submit
) Biomed Central 